\title{
Peptide nucleic acid (PNA) binding-mediated gene regulation
}

\author{
Gan WANG, Xiaoxin S XU \\ Institute of Environmental Health Sciences, Wayne State University, 2727 Second Avenue, Detroit, MI 48201, USA
}

\begin{abstract}
Peptide nucleic acids (PNAs) are synthetic oligonucleotides with chemically modified backbones. PNAs can bind to both DNA and RNA targets in a sequence-specific manner to form PNA/DNA and PNA/RNA duplex structures. When bound to double-stranded DNA (dsDNA) targets, the PNA molecule replaces one DNA strand in the duplex by strand invasion to form a PNA/DNA/PNA [or (PNA) 2 /DNA] triplex structure and the displaced DNA strand exists as a singlestranded D-loop. PNA has been used in many studies as research tools for gene regulation and gene targeting. The Dloops generated from the PNA binding have also been demonstrated for its potential in initiating transcription and inducing gene expression. PNA provides a powerful tool to study the mechanism of transcription and an innovative strategy to regulate target gene expression. An understanding of the PNA-mediated gene regulation will have important clinical implications in treatment of many human diseases including genetic, cancerous, and age-related diseases.
\end{abstract}

Keywords: peptide nucleic acids (PNAs), PNA binding, single-stranded D-loop, transcription initiation.

\section{Introduction}

Peptide nucleic acids (PNAs) are synthetic oligonucleotides with modified backbones[1]. In PNAs, the sugar backbone is replaced with peptide-like backbones (Fig 1). PNAs can bind to both DNA and RNA targets in a sequence-specific manner to form a Watson-Crick type PNA/ DNA and a PNA/RNA double helical structure. PNAs can also bind to double-stranded DNA (dsDNA) targets. In this case, the PNA molecule replaces one of the complimentary DNA strands by strand invasion and the displaced DNA strand then exists as a single-stranded D-loop at the PNA binding site[1-6]. When PNAs bind to homopurine/ homopyrimidine sequences, the PNA/DNA/PNA [or (PNA) ${ }_{2}$ /DNA] triple helix structures can be formed (Fig 2A). Studies suggest that the first PNA molecule binds with the DNA strand to form a Watson-Crick type duplex structure and the second PNA molecule binds to the DNA strand of the PNA/DNA duplex by Hoogsteen hydrogen bonds $[7,8]$ to form the (PNA) 2 /DNA triplex structure[1, 3, 911]. Most of the triplexes formed by PNAs are pyrimidine-motif triplex structures in which the pyrimidine-containing PNA molecule binds parallel to the purine-containing DNA strand of the PNA/DNA duplex to form the (PNA) ${ }_{2} / \mathrm{DNA}$ triplex structure[1, 9, 12, 13]. The (PNA) $)_{2}$ DNA triplex structure stabilizes both the bound PNAs and the

${ }^{*}$ Correspondence: Gan WANG

Tel: 313-964-8140, Fax: 313-577-0082;

E-mail: g.wang@wayne.edu single-stranded D-loop at the binding site[14].

PNAs have been used in a variety of research applications. One of the most common applications of the PNA technology is its antisense application in which the PNAs are designed to bind to messenger RNA (mRNA) targets to inhibit translation of the target genes[15-27]. PNAs are also used as probes for gene cloning and mutation detection[17, 28-30]. PNAs designed to bind to specific target gene sequences have been used in homologous recombination studies[31, 32]. In addition, PNAs designed as transcription factor decoy molecules have also been used for target gene induction[33].

The D-loops generated by PNA binding to dsDNA targets have been used to induce transcription and gene expression (Fig 2B). Early studies revealed that the PNA binding-generated D-loops could initiate transcriptions in vitro using T7 RNA polymerase[34]. The work of our laboratory demonstrated that the PNA binding-generated D-loops could initiate transcription in a HeLa nuclear extract in vitro transcription system and induce a GFP reporter gene expression in the CV-1 monkey kidney cells[35]. Most importantly, when PNAs designed to bind to the human $\gamma$ globin gene 5' untranslated region (5' UTR) sequence were used to treat the K562 human erythroleukemia cells, transcription of the endogenous $\gamma$-globin gene from the PNA binding sites was achieved[35]. These results suggest that PNA binding-mediated transcription may provide an innovative strategy to induce expression of specific target genes. It may also provide a novel approach to study gene modu- 


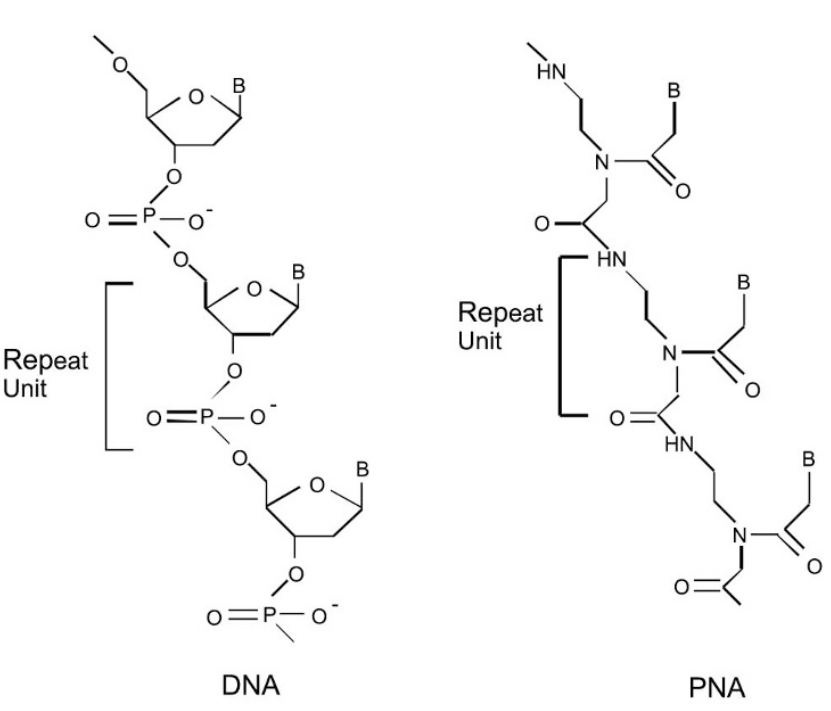

Fig 1. Structures of DNA and PNA oligonucleotides.

lating, transcription, and transcription regulation. Although the molecular mechanism of PNA binding-mediated transcription is relatively unclear, the results obtained from our recent studies provide some valuable information regarding the molecular basis of this process. We will discuss some of the progress that have been made in the PNA studies in the past few years and outline some of the difficulties that need to be overcome to bring the field forward.

\section{PNA binding}

PNAs can bind to both DNA and RNA targets in a sequence-specific manner to form duplex structures. Although the Watson-Crick duplex formation (anti-parallel duplex formation) is the preferred formation for the PNA bindings, parallel duplex structure can still be formed when PNAs bind to the target sequences[36].

The binding of PNAs to DNA and RNA targets is stronger than that of DNA/DNA or RNA/RNA bindings[1, 37]. This enhanced binding affinity is partially due to the uncharged property of the PNAs. Since PNAs are neutral in charge, the duplexes formed by PNA/DNA or PNA/RNA hybrids lack the electrostatic repulsion formed by DNA/ DNA or RNA/DNA duplexes, resulting in a very stable duplex formation even at a relatively high temperature and a very high binding affinity. For example, the melting temperature $\left(\mathrm{T}_{\mathrm{m}}\right)$ of a normal $\mathrm{dA}_{10}-\mathrm{dT}_{10}$ DNA hybrid is $23^{\circ} \mathrm{C}$ while the $\mathrm{T}_{\mathrm{m}}$ of a similar $\mathrm{dA}_{10}-\mathrm{dT}_{10} \mathrm{DNA} / \mathrm{PNA}$ hybrid is $86^{\circ} \mathrm{C}[1]$.

As a result of this high binding affinity, the binding specificity of PNAs, in general, is much higher than that of DNA/DNA or RNA/RNA bindings. Compared with the

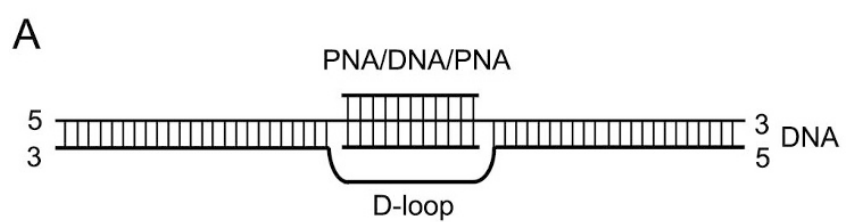

B

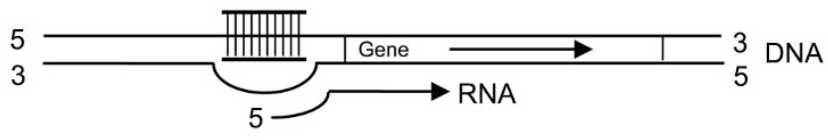

Fig 2. PNA binding-generated (PNA) $)_{2}$ DNA triplex and singlestranded D-loop structure $(\mathbf{A})$ and the transcription initiated from the D-loop site (B).

DNA/DNA or RNA/RNA bindings, any mismatches contained at the PNA binding sites will have greater impact on the stability of the duplexes formed by PNA/DNA and PNA/ RNA hybrids[1, 38-42]. For example, binding kinetics studies reveal that the binding affinity of the $\mathrm{T}_{5} \mathrm{CT}_{4}$ PNA to the $\mathrm{dA}_{10}$ target is 100 times lower than that of the $\mathrm{T}_{10}$ PNA binding to the same target[42]. For this reason, PNAs are often used as probes for identifying desired target gene sequences and for detecting single base mutations with high accuracy[17, 28-30, 43]. The lengths of PNAs used in the hybridization studies are also relatively shorter than the normal DNA or RNA probes because of this high binding affinity. In most of the hybridization studies, PNAs with lengths of 8-12 mer are sufficient to form strong duplex formation and to distinguish single base mutations [17, 28-30]. When long PNA molecules are used as probes in the hybridization, however, reduced specificities may result since the stronger binding affinity between the PNAs and the homologous non-target sequences may lead to an increased false positive identification rate for the desired target sequences.

\section{The effects of salts on PNA binding}

Although the binding of PNA with DNA and RNA is stronger, this binding process of PNAs to dsDNA targets can be influenced by the presence of salts. This is because the binding of PNAs to dsDNA targets needs to replace one of the complimentary DNA strands in the duplex and the presence of positively charged ions, especially at high concentrations, can inhibit the strand invasion process by preventing the interaction of PNA molecule with the DNA targets, resulting in ineffective binding[6]. In comparison, the binding of PNAs to single-stranded targets such as mRNA or single-stranded DNA targets is not affected by the presence of salts. For example, in our previous studies the presence of $150 \mathrm{mM} \mathrm{KCl}$, which is relevant to the physiological $\mathrm{K}^{+}$concentration, significantly inhibited the 
binding of PNA-1 to the target[35].

To enhance the binding ability of PNAs to the dsDNA targets in the presence of salts, several strategies have been explored. Incorporation of positively charged groups such as lysine and arginine into the PNA molecules has been demonstrated to lead to an enhanced PNA binding in the presence of high concentrations of salts[10, 34, 44, 45]. Conjugating a positively charged peptide has also been determined to lead to an increase in PNA binding, especially the PNA binding to dsDNA targets[22]. This is likely due to the positive charges contained in the PNA that help the PNA molecule compete with other positively charged ions for an interaction with the negatively charged DNA targets, resulting in enhanced PNA bindings in the presence of salts. Increasing target gene transcription has also lead to an increase in the PNA binding efficiency in the presence of high concentrations of salts[46]. The sequence context of the PNA binding targets may also contribute to the PNA binding inhibition in the presence of high concentrations of salts. In our earlier studies, we found that two different PNAs of slightly different sequences but targeting to the regions of the genome separated by only $6 \mathrm{bp}$ had quite different salt dependence[35]. Therefore, if the salt inhibition effect is observed in the chosen PNA targets, changing PNA binding sites to other positions may help overcome this inhibition effect.

\section{The stability of PNA}

In comparison to normal DNA and RNA oligonucleotides, PNAs are much more stable inside cells[47]. PNAs are very resistance to nuclease digestion since the nucleases cannot digest their altered backbones[48]. PNAs are also very resistant to protease digestion. Regular DNA and RNA oligonucleotides are very sensitive to the nuclease digestion. Inside cells, for example, the half-life for most of the unmodified DNA and RNA oligonucleotides is approximately $15 \mathrm{~min}$ or shorter. In contrast, PNAs are very stable inside cells. Studies indicate that PNAs are stable inside cells for at least $48 \mathrm{~h}[49]$. This extreme stability makes PNAs an ideal candidate for the antisense and antigene application.

\section{PNA delivery}

Although PNAs have many advantages in its application, some disadvantages have limited its application. One of the most important issues in the PNA application is its delivery efficiency. Unlike most of the DNA and RNA oligonucleotides, which can be easily delivered into cells through endocytosis, PNAs are poorly penetrated through the cell membrane[9, 50]. This is partially due to its uncharged property. Since the cell membrane carries nega- tive charges, positively charged molecules such as normal DNA and RNA oligonucleotides can be attracted to the cell membrane, resulting in penetration of the oligonucleotides through the cell membrane by endocytosis. In contrast, uncharged PNA molecules cannot be attracted to the membrane, resulting in poor penetration through the membrane by this endocytotic process.

To enhance the efficiency in PNA delivery, many strategies have been explored. Since a positive charge will enhance the attraction of molecules to cell membrane, some studies have been attempted by incorporating positively charged residues such as lysine and arginine to the PNA molecules to enhance the PNA delivery efficiency[51]. Studies have also been done using ligands to enhance the attachment of PNAs to the cell membrane[15, 16, 18 ,20, 21, 52-59]. For example, PNAs have been conjugated with short peptide sequences, to enhance the PNA delivery efficiency[15, 53]. PNAs conjugated with other ligands such as antibodies or steroids have also been used to increase the PNA delivery efficiency[16, 52, 54, 60-64].

\section{PNA binding-induced transcription}

The possibility of using the PNA binding-generated Dloops to induce gene-specific transcription and gene expression has been studied both in vitro and in vivo [34, 35, 65]. The work reported by Mollegard et al indicated that a PNA $T_{10}$ binding-generated D-loop was able to induce transcription from the D-loop site in both a T7 RNA polymerase -based in vitro transcription system and a rat nuclear extract in vitro transcription system[34]. In that work, the transcription activity of the $\mathrm{T}_{10}$ PNA-generated D-loop was determined by comparison with the transcription activity initiated from an $E$. coli lacUV5 promoter. The in vitro transcription results indicated that the transcription activity of the $\mathrm{T}_{10}$ PNA-generated D-loop is comparable to that of the lacUV5 promoter[34].

In our previous studies, we determined the transcription initiated from PNA binding-generated D-loops using two PNAs designed to bind to the human $\gamma$-globin gene -280 region, one with a 10 -base target sequence and the second with a 12-base target sequence. The results obtained from our in vitro transcription studies indicated that both PNAs can induce transcription in a HeLa nuclear extract in vitro transcription system; the D-loop generated by the 12-mer PNA showed a much stronger transcription activity than that by the 10-mer PNA[35]. We also studied the abilities of these PNAs to induce gene expression using a plasmid construct that carries both PNA binding site sequences with a promoterless green fluorescent protein (GFP) gene[35]. When the PNA-bound plasmid was transfected into the CV-1 monkey kidney cells by microinjection, expression of the GFP protein was detected in 
the transfected cells[35]. This result suggests that the PNA binding-generated D-loops could not only induce transcription in vitro but also induce expression of target gene in vivo, revealing a great potential of PNAs in inducing genespecific expression in living cells.

The possibility of utilizing PNAs to induce expression of endogenous genes was also investigated in our previous studies using the PNAs designed to bind to the human $\gamma$-globin gene 5' UTR sequence[35]. When the K562 human erythroleukemia cells were treated with the PNAs for two days, specific transcripts initiated from the PNA binding sites were detected from the treated K562 cells; however, these transcripts were not detected in the untreated K562 cells. Interestingly, the results obtained from our studies indicate that the PNA treatment also enhances the transcription of the endogenous $\gamma$-globin gene from its natural promoter, suggesting that a transcription event occurring at the nearby region might result in an altered chromatin structure, leading to an increased transcription activity from its promoter. These results suggest that the PNA bindinggenerated D-loops could indeed provide an innovative strategy in inducing desired target gene expression, indicating the important clinical potentials of PNAs in inducing expression of some therapeutic-important genes.

\section{The PNA length requirement for inducing tran- scription from the PNA binding sites}

The results of our studies and the data published by others suggest that the D-loops generated by the PNA bindings could be used as artificial promoters to initiate transcription[34, 35, 66]. The lengths of the PNA required for the PNA binding-mediated transcription, however, were not determined in these studies. To determine the optimal PNA length requirement for inducing transcription from the PNA binding sites, we have designed and synthesized a series of PNAs ( 8 mer-20 mer) that bind to a $20 \mathrm{bp}$ homopurine/homopyrimidine site in a GFP reporter genecontaining plasmid construct. The transcription activity initiated from the PNA binding-generated D-loops was then determined[65]. The results obtained from our in vitro transcription assay indicated that the transcription activity was detected with PNAs of 14 mer to 20 mer in length. The highest transcription activity, however, was detected when the PNAs of 16 mer and 18 mer PNA were preincubated with the plasmid DNA. To determine the PNA length requirement for inducing gene expression in vivo, various lengths of the PNA-bound plasmid DNA were transfected into human normal fibroblast (NF) cells. The GFP gene expression was then monitored with results indicating high levels of GFP expression by the 16 mer and 18 mer-PNA bound plasmid DNA. These results suggest that PNAs with lengths of 16-18 mer are more effective in inducing transcription and target gene expression.

\section{The basal transcription components involved in the PNA binding-induced transcription}

Although PNA binding-generated D-loops have been demonstrated for their abilities in inducing transcriptions $[34,35,65]$, the molecular mechanism for PNA bindinginduced transcription has not been established. Detection of PNA binding-induced GFP expression in mammalian cells suggests that a class II nuclear gene transcription may be involved in the PNA binding-induced transcription. To define the transcription factors involved in the PNA binding-mediated transcription, we have recently studied the PNA binding-mediated transcription in vitro using a depleted HeLa nuclear extract system (Wang, unpublished data). The basal transcription factors, including TFIIB, TFIID, TFIIE, and TFIIH, and the RNA polymerase II, were individually depleted in the HeLa nuclear extract by immuno-precipitation. The PNA binding-mediated transcription was then studied in both the immuno-depleted HeLa nuclear extracts and the extracts supplemented with purified individual basal transcription factors. The results obtained from our in vitro transcription experiments indicate that the basal transcription factors TFIID and TFIIH and the RNA polymerase II are essential for the PNA binding-induced transcription. The basal transcription factors TFIIB and TFIIE, however, are not essential for the PNA binding-mediated transcription. This result suggests that the class II gene transcription is indeed involved in the PNA binding-induced transcription process. Since the PNA binding-generated D-loops are structurally different from the class II gene promoters, it remains unclear as to how the basal transcription factors, such as the TFIID, can recognize the PNA binding-generated D-loops and initiate the transcription from the D-loop sites. Further studies are needed to determine the interactions between the PNA binding-generated D-loops and the basal transcription factors and the roles of these interactions in the initiation of transcription.

\section{Conclusion}

PNA binding-mediated transcription provides an innovative strategy to induce gene expression. This strategy will have both scientific importance and clinical relevance. The PNA binding-mediated transcription provides a powerful method to study the molecular mechanism of transcription initiation and gene regulation. The PNA binding mediated gene expression will also have important clinical implications in treatment of many human disease such as cancer and genetic diseases. However, some difficulty 
exists in the technology that has limited its applications. The molecular mechanism of PNA binding-induced transcription needs to be established to provide a better understanding for the molecular basis of this important approach. Once these issues are resolved, the vast potential of this novel technology may be realized in the treatment of many human diseases and possible other applications.

\section{REFERENCES}

1 Nielsen PE, Egholm M, Berg RH, Buchardt O. Sequence-selective recognition of DNA by strand displacement with a thyminesubstituted polyamide. Science 1991; 254:1497-500.

2 Kurakin A, Larsen HJ, Nielsen PE. Cooperative strand displacement by peptide nucleic acids (PNA). Chem \& Biol 1998; 5:81-9.

3 Lohse J, Dahl O, Nielsen PE. Double duplex invasion by peptide nucleic acid: a general principle for sequence-specific targeting of double-stranded DNA. Proc Natl Acad Sci USA 1999; 96:11804-8.

4 Lesignoli E, Germini A, Corradini R, et al. Recognition and strand displacement of DNA oligonucleotides by peptide nucleic acids (PNAs). High-performance ion-exchange chromatographic analysis. J Chromatogr A 2001; 922:177-85.

5 Nielsen PE, Egholm M. Strand displacement recognition of mixed adenine-cytosine sequences in double stranded DNA by thymine-guanine PNA (peptide nucleic acid). Bioorg Med Chem 2001; 9:2429-34.

6 Demidov VV, Frank-Kamenetskii MD. Sequence-specific targeting of duplex DNA by peptide nucleic acids via triplex strand invasion. Methods 2001; 23:108-22.

7 Broitman SL, Im DD, Fresco JR. Formation of the triple-stranded polynucleotide helix, poly(AAU). Proc Natl Acad Sci USA 1987; 84:5120-4.

8 Sun JS, Helene C. Oligonucleotide-directed triple-helix formation. Current Opinion Struct Biol 1993; 3:345-56.

9 Hanvey JC, Peffer NJ, Bisi JE, et al. Antisense and Antigene Properties of Peptide Nucleic Acids. Science 1992; 258:1481-5.

10 Bentin T, Larsen HJ, Nielsen P E. Combined triplex/duplex invasion of double-stranded DNA by "Tail-Clamp" Peptide nucleic Acid Biochemistry 2003; 42:13987-95.

11 Bentin T, Nielsen PE. Superior duplex DNA strand invasion by acridine conjugated peptide nucleic acids. J Am Chem Soc 2003; 125:6378-9.

12 Knudsen H, Nielsen PE. Antisense properties of duplex- and triplex-forming PNAs. Nucleic Acids Res 1996; 24:494-500.

13 Demidov VV, Cherny DI, Kurakin AV, et al. Stability of peptide nucleic acids in human serum and cellular extracts. Nucleic Acids Res 1994; 22:5218-22.

14 Krupnik OV, Guscho Y, Sluchanko K, Nielsen P, Lazurkin Y. Thermodynamics of the melting of PNA(2)/DNA triple helices. J Biomol Struct Dyn 2001; 19:535-42.

15 Aldrian-Herrada G, Desarmenien MG, Orcel H, et al. A peptide nucleic acid (PNA) is more rapidly internalized in cultured neurons when coupled to a retro-inverso delivery peptide. The antisense activity depresses the target mRNA and protein in magnocellular oxytocin neurons. Nucleic Acids Res 1998; 26: 4910-6.

16 Penichet ML, Kang YS, Pardridge WM, Morrison SL, Shin SU. An antibody-avidin fusion protein specific for the transferrin receptor serves as a delivery vehicle for effective brain targeting: initial applications in anti-HIV antisense drug delivery to the brain. J Immunol 1999; 163:4421-6.

17 Tyler BM, Jansen K, McCormick DJ, et al. Peptide nucleic acids targeted to the neurotensin receptor and administered i.p. cross the blood-brain barrier and specifically reduce gene expression. Proc Natl Acad Sci USA1999; 96:7053-8.

18 Pooga M, Soomets U, Hallbrink M, et al. Cell penetrating PNA constructs regulate galanin receptor levels and modify pain transmission in vivo. Nat Biotech 1998; 16:857-61.

19 Efferth T, Fabry U, Osieka R. Leptin contributes to the protection of human leukemia cellls from cisplatinum cytoxicity. Anticancer Res 2000; 20:2441-546.

20 Cutrona G, Carpaneto EM, Uliv M, et al. Effects in loive cells of a c-myc anti-gene PNA linked to a localization signal. Nature Biotechnology 2000; 18:300-3.

21 Scarfi S, Giovine M, Gasparini A, et al. Modified peptide nucleic acids are internalized in mouse macrophages RAW 264.7 and inhibit inducible nitric oxide synthase. FEBS Letters 1999; 451: 264-8.

22 Kaihatsu K, Braasch DA, Cansizoglu A, Corey DR. Enhanced strand invasion by peptide nucleic acid-peptide conjugates. Biochemistry 2002; 41:11118-25.

23 Komiyama M, Ye S, Liang X, et al. PNA for one-base differentiating protection of DNA from nuclease and its use for SNPs detection. J Am Chem Soc 2003; 125:3758-62.

24 Van Rossenberg SM, Sliedregt-Bol KM, Prince P, et al. A targeted Peptide nucleic Acid to down-regulate mouse microsomal triglyceride transfer protein expression in hepatocytes. Bioconjug Chem 2003; 14:1077-82.

25 Heckl S, Pipkorn R, Waldeck W, et al. Intracellular visualization of prostate cancer using magnetic resonance imaging. Cancer Res 2003; 63:4766-72.

26 Nielsen PE. Antisense peptide nucleic acids. Curr Opin Mol Ther 2000; 2:282-7.

27 Zhao X, Kaihatsu K, Corey DR. Inhibition of transcription by bisPNA-peptide conjugates. Nucleosides Nucleotides Nucleic Acids 2003; 22:535-46.

28 Orum H, Nielsen PE, Egholm M, et al. Single base pair mutation analysis by PNA directed PCR clamping. Nucleic Acids Res 1993; 21:5332-6.

29 Sotlar K, Escribano L, Land O, et al. One-step detection of c-kit point mutations using peptide nucleic acid-mediated polymerase chain reaction clamping and hybridization probes. Am J Pathol 2003; 162:737-46.

30 Ye S, Liang X, Yamamoto Y, et al. Simultaneous detection of multiple single nucleotide polymorphism by single-strand-specific nuclease and PNA probe. Nucleic Acids Res 2003; Suppl, 3:185-6.

31 Faruqi AF, Egholm M, Glazer PM. Peptide nucleic acid-targeted mutagenesis of a chromosomal gene in mouse cells. Proc Natl Acad Sci USA 1998; 95:1398-403.

32 Rogers FA, Vasquez KM, Egholm M, Glazer PM. Site-directed recombination via bifunctional PNA-DNA conjugates. Proc Natl Acad Sci USA 2002; 99:16695-700.

33 Borgatti M, Lampronti I, Romanelli A, et al. Transcription factor decoy molecules based on a peptide nucleic acid (PNA)DNA chimera mimicking Sp1 binding sites. J Biol Chem 2003; 278:7500-9. 
34 Mollegaard NE, Buchardt O, Egholm M, Nielsen PE. Peptide nucleic acid.DNA strand displacement loops as artificial transcription promoters. Proc Natl Acad Sci USA 1994; 91:3892-5.

35 Wang G, Xu X, Pace B, et al. Peptide nucleic acid (PNA) binding-mediated induction of human $\gamma$-globin gene expression. Nucleic Acids Res 1999; 27:2806-13.

36 Egholm M, Buchardt O, Christensen L, et al. PNA hybridizes to complementary oligonucleotides obeying the Watson-Crick hydrogen-bonding rules. Nature 1993; 365:490-2.

37 Chakrabarti MC, Schwarz FP. Thermal stability of PNA/DNA and DNA/DNA duplexes by differential scanning calorimetry. Nucleic Acids Res 1999; 27:4801-6.

38 Nielsen PE, Egholm M, Buchardt O. Sequence-specific transcription arrest by peptide nucleic acid bound to the DNA template strand. Gene 1994; 149:139-45.

39 Nielsen PE, Egholm M, Berg RH, Buchardt O. Sequence specific inhibition of DNA restriction enzyme cleavage by PNA. Nucleic Acids Res 1993; 21:197-200.

40 Taylor RW, Chinnery PF, Turnbull DM, Lightowlers RN. Selective inhibition of mutant human mitochondrial DNA replication in vitro by peptide nucleic acids. Nature Genetics 1997; 15:212-5.

41 Doyle DF, Braasch DA, Simmons CG, Janowski BA, Corey D R. Inhibition of gene expression inside cells by peptide nucleic acids: effect of mRNA target sequence, mismatched bases, and PNA length. Biochemistry 2001; 40:53-64.

42 Demidov VV, Yavnilovich MV, Belotserkovskii BP, FrankKamenetskii MD, Nielsen PE. Kinetics and mechanism of polyamide ("peptide") nucleic acid binding to duplex DNA. Proc Natl Acad Sci USA 1995; 92:2637-41.

43 Basile A, Giuliani A, Pirri G, Chiari M. Use of peptide nucleic acid probes for detecting DNA single-base mutations by capillary electrophoresis. Electrophoresis 2002; 23:926-9.

44 Smulevitch SV, Simmons CG, Norton JC, Wise TW, Corey DR. Enhancement of strand invasion by oligonucleotides through manipulation of backbone charge. Nat Biotechnol 1996; 14:1700-4.

45 Kaihatsu K, Shah RH, Zhao X, Corey DR. Extending Recognition by Peptide Nucleic Acids (PNAs): Binding to Duplex DNA and Inhibition of Transcription by Tail-Clamp PNA-Peptide Conjugates. Biochemistry 2003; 42:13996-4003.

46 Larsen HJ, Nielsen PE. Transcription-mediated binding of peptide nuclei acid (PNA) to double-stranded DNA: sequence-specific suicide transcription. Nucleic Acids Res 1996; 24:458-63.

47 Demidov VV, Potaman VN, Frank-Kamenetskii MD, et al. Electron microscopy mapping of oligopurine tracts in duplex DNA by peptide nucleic acid targeting. Biochemical pharmacology 1994; 48:1310-3.

48 Borgatti M, Romanelli A, Saviano M, et al. Resistance of decoy PNA-DNA chimeras to enzymatic degradation in cellular extracts and serum. Oncol Res 2003; 13:279-87.

49 McMahon BM, Mays D, Lipsky J, et al. Pharmacokinetics and tissue distribution of a peptide nucleic acid after intravenous administration. Antisense Nucleic Acid Drug Dev 2002; 12:6570 .

50 Pardridge WM, Boado RJ, Kang YS. Vector-mediated delivery of a polyamide ('petide') nucleic acid analogue through the bloodbrain barrier in vivo. Proc Natl Acad Sci USA 1995; 92:5592-6.

51 Filipovska A, Eccles MR, Smith RA, Murphy MP. Delivery of antisense peptide nucleic acids (PNAs) to the cytosol by disulphide conjugation to a lipophilic cation. FEBS Lett 2004; 556:180-6.

52 Boado RJ, Tsukamoto H, Pardridge WM. Drug delivery of antisense molecules to the brain for treatment of Alzheimer's disease and cerebral AIDS. J Pharm Sci 1998; 87:1308-15.

53 Braun K, Peschke P, Pipkorn R, et al. A biological transporter for the delivery of peptide nucleic acids (PNAs) to the nuclear compartment of living cells. J Mol Biol 2002; 318:237-43.

54 Rebuffat AG, Nawrocki AR, Nielsen PE, et al. Gene delivery by a steroid-peptide nucleic acid conjugate. FASEB J 2002; 16:1426-8.

55 Basu S, Wickstrom E. Synthesis and characterization of a peptide nucleic acid conjugated to a D-peptide analog of insulin-like growth factor 1 for increased cellular uptake. Bioconj Chem 1997; 8:481-8.

56 Bertrand JR, Sumbatyan N, Malvy C. Covalent coupling of a PIM-1 oncogene targeted PNA with an antennapedia derived peptide. Nucleosides Nucleotides Nucleic Acids 2003; 22:1611-3.

57 Chinnery PF, Taylor RW, Diekert K, et al. Peptide nucleic acid delivery to human mitochondria. Gene Therapy 1999; 6:1919-28.

58 Gait MJ. Peptide-mediated cellular delivery of antisense oligonucleotides and their analogues. Cell Mol Life Sci 2003; 60:844-53.

59 Simmons CG, Pitts AE, mayfield LD, Shay JW, Corey DR. Synthesis and permeability of PNA-peptide conjugates. Bioorg Med Chem Lett 1997; 7:3001-7.

60 Obara K, Ishihara T, Akaike T, Maruyama A. Protein/oligonucleotide conjugates as a cell specific PNA carrier. Nucleic Acids Res 2001; Suppl, 1:217-8.

61 Braasch D A, Corey DR. Lipid-mediated introduction of peptide nucleic acids into cells. Methods Mol Biol 2002; 208:21123.

62 Folini M, Berg K, Millo E, et al. Photochemical internalization of a peptide nucleic acid targeting the catalytic subunit of human telomerase. Cancer Res 2003; 63:3490-4.

63 Yuan X, Ma Z, Zhou W, et al. Lipid-mediated delivery of peptide nucleic acids to pulmonary endothelium. Biochem Biophys Res Commun 2003; 302:6-11.

64 Koppelhus U, Nielsen PE. Cellular delivery of peptide nucleic acid (PNA). Adv Drug Deliv Rev 2003; 55:267-80.

65 Wang G, Jing K, Balczon R, Xu X. Defining the peptide nucleic acids (PNA) length requirement for PNA binding-induced transcription and gene expression. J Mol Biol 2001; 313:933-40.

66 Mollegaard NE, Nielsen PE. In vitro transcription from peptide nucleic acid/DNA strand displacement loops. Methods Mol Biol 2002; 208:249-56. 